\title{
Remodeling the effective one-body formalism in post-Minkowskian gravity
}

\author{
Poul H. Damgaard $\oplus^{1}$ and Pierre Vanhove $\oplus^{2,3}$ \\ ${ }^{1}$ Niels Bohr International Academy Niels Bohr Institute, University of Copenhagen \\ Blegdamsvej 17, DK-2100 Copenhagen $\varnothing$, Denmark \\ ${ }^{2}$ Université Paris-Saclay, CNRS, CEA, Institut de physique théorique, 91191 Gif-sur-Yvette, France \\ ${ }^{3}$ National Research University Higher School of Economics, Myasnitskaya Ulitsa, 20, Moscow 101000, \\ Russian Federation
}

(Received 30 August 2021; accepted 6 October 2021; published 12 November 2021)

\begin{abstract}
The effective one-body formalism of the gravitational two-body problem in general relativity is reconsidered in the light of recent scattering amplitude calculations. Based on the kinematic relationship between momenta and the effective potential, we consider an energy-dependent effective metric describing the scattering in terms of an effective one-body problem for the reduced mass. The identification of the effective metric simplifies considerably in isotropic coordinates when combined with a redefined angular momentum map. While the effective energy-dependent metric as expected is not unique, solutions can be chosen perturbatively in the post-Minkowskian expansion without the need to introduce nonmetric corrections. By a canonical transformation, our condition maps to the one based on the standard angular momentum map. Expanding our metric around the Schwarzschild solution we recover the solution based on additional nonmetric contributions.
\end{abstract}

DOI: 10.1103/PhysRevD.104.104029

\section{INTRODUCTION}

Recent advances in the scattering amplitude-based approach to the post-Minkowskian expansion of classical general relativity [1-29] have demonstrated that this new approach holds the promise of significantly changing the efficiency of computations in general relativity. The input from scattering amplitude calculations is increasing fast. At this point, full third-order post-Minkowskian amplitude calculations of the scattering of two massive objects are now available [7,14-16,20,29], and the first results for fourth post-Minkowskian order have already appeared [17]. This amplitude-based approach generically computes one observable; the scattering angle in what we can call the hyperbolic regime of the two-body problem in gravity. Although of interest in themselves, eventually these results should be used to predict gravitational waveforms and other observables associated with two massive objects bound to each other. One strategy for going from the scattering regime to the bound-state regime is based on the effective one-body (EOB) formalism [30,31], suitably adapted from postNewtonian to post-Minkowskian formulations [1-3]. So, hugely successful based on post-Newtonian computations,

Published by the American Physical Society under the terms of the Creative Commons Attribution 4.0 International license. Further distribution of this work must maintain attribution to the author(s) and the published article's title, journal citation, and DOI. Funded by SCOAP ${ }^{3}$. it seems timely to revisit this EOB approach and explore both its flexibility and its power of prediction.

The aim of this paper is to gather known results up to third post-Minkowskian order in Newton's constant $G_{N}$ and include them in the most compact manner in a postMinkowskian version of the EOB formalism. The choice of isotropic coordinates is crucial for simplicity. Interestingly, once in isotropic coordinates, we find that the simplest approach is to not expand around the probe limit of the two-body problem, which would correspond to motion in the background metric of a Schwarzschild black hole. The way to achieve this is to enlarge the notion of the effective metric so that it becomes energy dependent. This possibility appears to be intuitively appealing and understandable for the gravitational scattering of two massive objects which, due to the nonlinearities of general relativity beyond the probe limit, create backreactions that depend on energy and momentum. Although the effective metric itself depends on the energy, we can still impose the standard quadratic mass-shell condition; we will find the correct map that describes the gravitational scattering of two massive objects such that the scattering angle deduced from that metric coincides with the one computed from the post-Minkowskian expansion of the full theory. Choosing an angular momentum map that differs from the one conventionally used [30,31] connects most straightforwardly to the scattering amplitude-based approach to general relativity, and we end up describing the reduced problem in terms of a massive object in an effective metric that only reduces to the Schwarzschild metric in the probe 
limit. Moreover, as we will demonstrate, the motion is entirely described by this metric without, at least up to the present order, introduction of correction terms of the nonmetric kind. By a canonical transformation, we also recover the condition based on the standard angular momentum map, without the need to include nonmetric corrections. Expanding our metric around the Schwarzschild metric can rephrase the solution in terms of the combination of a Schwarzschild metric plus additional nonmetric terms, finding complete agreement with the solution given in that form by Damour in Ref. [3].

\section{POST-MINKOWSKIAN KINEMATICS AND THE EFFECTIVE METRIC}

While the EOB formalism is a standard tool for the gravitational wave physics community [30-34], it is not widely known in the particle physics community. Since the aim of this paper is to explore some of the consequences of calculating classical general relativity observables with modern scattering-amplitude methods, we begin this section with an elementary introduction to the EOB formalism, phrased in a manner that may be more accessible to particle physicists.

We begin by considering free-particle kinematics in Minkowskian space. The aim is to describe the dynamics of two masses $m_{1}$ and $m_{2}$ moving with relative velocity

$$
v \equiv|\vec{v}|=\left|\vec{v}_{1}-\vec{v}_{2}\right|,
$$

in terms of a reduced mass

$$
\mu \equiv \frac{m_{1} m_{2}}{m_{1}+m_{2}},
$$

moving with the same velocity $v$. It is convenient to introduce the total mass $M \equiv m_{1}+m_{2}$ so that $\mu=$ $m_{1} m_{2} / M$. In terms of the original relativistic kinematics, the Lorentz contraction factor is

$$
\gamma=\frac{E^{2}-m_{1}^{2}-m_{2}^{2}}{2 m_{1} m_{2}}=\frac{p_{1} \cdot p_{2}}{m_{1} m_{2}},
$$

with $p_{i}$ being the two momenta and where $E$ is the total energy. Solving Eq. (3) for $E$ in this frame, we have the relation

$$
E=M \sqrt{1+2 \nu(\gamma-1)}
$$

where

$$
\nu \equiv \frac{m_{1} m_{2}}{\left(m_{1}+m_{2}\right)^{2}}=\frac{\mu}{M} .
$$

Denoting by $\mathcal{E}_{\text {eff }}=\mu \gamma$ the energy of the reduced mass $\mu$, this leads to the relation

$$
H=E=M \sqrt{1+2 \nu\left(\frac{\mathcal{E}_{\mathrm{eff}}}{\mu}-1\right)} .
$$

This is the energy map.

To relate the corresponding magnitude of the threemomentum $p_{\text {eff }}=\left|\vec{p}_{\text {eff }}\right|$ of the reduced mass to the center-of-mass momentum $p_{\infty}$ of the two masses, we use free relativistic kinematics with $\vec{p}_{\text {eff }}=\mu \gamma \vec{v}$ and $\gamma=1 / \sqrt{1-\vec{v}^{2}}$. This gives

$$
\left(\frac{p_{\mathrm{eff}}}{\mu}\right)^{2}=\frac{\left(E^{2}-\left(m_{1}+m_{2}\right)^{2}\right)\left(E^{2}-\left(m_{1}-m_{2}\right)^{2}\right)}{4 m_{1}^{2} m_{2}^{2}},
$$

which is easily compared to the center-of-mass momentum $p_{\infty}$

$$
p_{\infty}^{2}=\frac{\left(E^{2}-\left(m_{1}+m_{2}\right)^{2}\right)\left(E^{2}-\left(m_{1}-m_{2}\right)^{2}\right)}{4 E^{2}},
$$

yielding

$$
\frac{p_{\mathrm{eff}}}{\mu}=\frac{p_{\infty} E}{m_{1} m_{2}} .
$$

This is the momentum map.

Finally, we wish to relate the angular momentum $J_{\text {eff }}$ of the reduced mass to the angular momentum $J$ of the two-particle system. We first choose to do this by insisting that impact parameter $b$ remains fixed. This is in contradistinction to the conventionally used prescription of [30] where, instead, angular momentum is kept fixed. We find our chosen relation more convenient for the following analysis because it more directly connects with the expression for the scattering angle we obtain from the two-body problem. The possibility of fixing $b$ instead of $J$ has been mentioned in Ref. [35] but not pursued there (see also Ref. [36] for a related discussion). Fixing $b$, we get

$$
b=\frac{J}{p_{\infty}}=\frac{J_{\mathrm{eff}}}{p_{\mathrm{eff}}} \Rightarrow J_{\mathrm{eff}}=J \frac{p_{\mathrm{eff}}}{p_{\infty}}=J \frac{E}{M} .
$$

This is our angular momentum map. We shall later show how to obtain the same results based on the conventional angular momentum map where, instead, one equates $J$ with $J_{\text {eff. }}$ This will involve a canonical transformation, thus leaving physics invariant.

\section{THE EFFECTIVE METRIC}

So far, we have not considered interactions. One important lesson from the scattering-amplitude approach to gravitational scattering in general relativity is that at least up to, and including, third post-Minkowskian order, there exists, in isotropic coordinates, a very simple relationship between center-of-mass momentum $p$ and the effective classical potential $V_{\text {eff }}(r, p)$ of the form $[7,10,11]$ 


$$
p^{2}=p_{\infty}^{2}-V_{\mathrm{eff}}(r, E)
$$

where, in $D=4$ dimensions,

$$
V_{\mathrm{eff}}(r, E)=-\sum_{n=1}^{\infty} f_{n}\left(\frac{G_{N} M}{r}\right)^{n} .
$$

The coefficients $f_{i}$ are deduced from the scattering angle

$$
\chi=G_{N} \chi_{1}+G_{N}^{2} \chi_{2}+G_{N}^{3} \chi_{3}+O\left(G_{N}^{4}\right),
$$

extracted from scattering-amplitude calculations orderby-order in the coupling $G_{N}$ as shown. Up to third post-Minkowskian order, the $f_{i}$-coefficients extracted from the amplitude computations read [28]

$$
\begin{aligned}
& f_{1}=2\left(2 \gamma^{2}-1\right) \frac{\mu^{2} M}{E}, \\
& f_{2}=\frac{3\left(5 \gamma^{2}-1\right)}{2} \frac{\mu^{2} M}{E},
\end{aligned}
$$

$$
\begin{aligned}
f_{3}= & -\mu^{2}\left(-\frac{3}{2} \frac{\left(2 \gamma^{2}-1\right)\left(5 \gamma^{2}-1\right)}{\gamma^{2}-1}+2 \frac{12 \gamma^{4}-10 \gamma^{2}+1}{\gamma^{2}-1} \frac{E}{M}\right) \\
& -\frac{2}{3} \frac{\mu^{2} \nu M}{E}\left(2 \gamma\left(14 \gamma^{2}+25\right)-\frac{\left(1-2 \gamma^{2}\right)^{2}}{\left(\gamma^{2}-1\right)^{2}}\left(8-5 \gamma^{2}\right) \sqrt{\gamma^{2}-1}+\left(\frac{6\left(4 \gamma^{4}-12 \gamma^{2}-3\right)}{\sqrt{\gamma^{2}-1}}-\frac{\left(6 \gamma^{3}-9 \gamma\right)\left(1-2 \gamma^{2}\right)^{2}}{\left(\gamma^{2}-1\right)^{2}}\right) \operatorname{arccosh}(\gamma)\right),
\end{aligned}
$$

$$
g_{\mathrm{eff}}^{\alpha \beta} \partial_{\alpha} \mathcal{S} \partial_{\beta} \mathcal{S}=\mu^{2}
$$

including all classical terms that contribute to this order. At fourth post-Minkowskian order, radiation must be taken into account and it is not yet obvious how this may affect, perturbatively in $G_{N}$, the order-by-order determination of the coefficients $f_{i}$.

Our aim now is to provide an effective one-body metric $g_{\mu \nu}^{\text {eff }}$ for the reduced-mass problem that reproduces the scattering angle computed from the expression of Eq. (27). Even if we specify isotropic coordinates it will quickly become clear that such an effective metric $g_{\mu \nu}^{\text {eff }}$ is not unique and part of our present purpose is therefore to explore the most optimal choice.

A general parametrization of $g_{\mu \nu}^{\text {eff }}$ can be provided by

$d s_{\mathrm{eff}}^{2}=A(r) d t^{2}-B(r)\left(d r^{2}+r^{2}\left(d \theta^{2}+\sin ^{2} \theta d \varphi^{2}\right)\right)$,

where $A(r)$ and $B(r)$ are so far undetermined functions of $r$. Because of the large set of coordinate transformations that are permissible within the choice of isotropic coordinates, we parametrize the solutions employing the ansatz

$$
A(r)=\left(\frac{1-h(r)}{1+h(r)}\right)^{2} ; \quad B(r)=(1+h(r))^{4} .
$$

In the limit $\nu \rightarrow 0$, we expect this effective metric to approach the Schwarzschild metric which in isotropic coordinates corresponds to

$$
h(r) \rightarrow \frac{G_{N} M}{2 r} .
$$

One standard method for computing the scattering angle in such an external metric is to determine the principal function $\mathcal{S}$ of the associated Hamilton-Jacobi equation
Because of conservation of the energy $\mathcal{E}_{\text {eff }}$ and angular momentum $J_{\text {eff }}$, and considering the motion in the orbital equatorial plane $\theta=\pi / 2$, we use the standard separated ansatz

$$
\mathcal{S}(r, t, \varphi)=\mathcal{E}_{\mathrm{eff}} t+J_{\mathrm{eff}} \varphi+W(r)
$$

to obtain

$$
\frac{\mathcal{E}_{\mathrm{eff}}^{2}}{A(r)}-\frac{J_{\mathrm{eff}}^{2}}{B(r) r^{2}}-\frac{1}{B(r)}\left(\frac{d W(r)}{d r}\right)^{2}=\mu^{2}
$$

and hence the scattering angle

$$
\frac{\chi}{2}=J_{\text {eff }} \int_{r_{m}}^{\infty} \frac{d r}{r^{2}} \frac{1}{\sqrt{\frac{B(r)}{A(r)} \mathcal{E}_{\text {eff }}^{2}-\frac{J_{\text {eff }}^{2}}{r^{2}}-B(r) \mu^{2}}}-\frac{\pi}{2},
$$

where $r_{m}$ is the distance of the closest radial approach in the scattering. This quantity is not independent and follows from the other parameters of the expression (23) through the condition

$p_{r}=\sqrt{\frac{B(r)}{A(r)} \mathcal{E}_{\mathrm{eff}}^{2}-\frac{J_{\mathrm{eff}}^{2}}{r^{2}}-B(r) \mu^{2}}=0 \quad$ at $r=r_{m}$.

Insisting on the angular momentum map of Eq. (10) and inserting also the momentum map (9), we can rewrite this as 


$$
\frac{\chi}{2}=b \int_{r_{m}}^{\infty} \frac{d r}{r^{2}} \frac{1}{\sqrt{\frac{B(r)}{A(r)} \frac{\mathcal{E}_{\text {eff }}^{2}}{p_{\text {eff }}}-\frac{b^{2}}{r^{2}}-\frac{B(r) \mu^{2}}{p_{\text {eff }}^{2}}}}-\frac{\pi}{2} .
$$

It is important to stress that we are employing momentum and angular momentum maps that were naturally provided at Minkowskian infinity and which are now taken to hold also in the presence of interactions. To fix, order-by-order in the coupling $G_{N}$, we compare the so far unknown functions $A(r)$ and $B(r)$ with the expression for the scattering angle obtained from the kinematic relation (11). This provides us with an alternative form of the radial action $W$ and hence

$$
\frac{\chi}{2}=-\int_{\hat{r}_{m}}^{\infty} d r \frac{\partial p_{r}}{\partial J}-\frac{\pi}{2},
$$

where, after using $p_{r}^{2}=p^{2}-\frac{J^{2}}{r^{2}}$ and substituting Eq. (11), we obtain

$$
\frac{\chi}{2}=b \int_{\hat{r}_{m}}^{\infty} \frac{d r}{r^{2}} \frac{1}{\sqrt{1-\frac{b^{2}}{r^{2}}-\frac{V_{\text {eff }}(r, E)}{p_{\infty}^{2}}}}-\frac{\pi}{2} .
$$

Because the two expressions (25) and (27) are so similar in form, we will now impose the strong requirement of the two integrands being equal. From the equality of the integrands, it follows that $r_{m}=\hat{r}_{m}$ since the condition $p_{r}=0$ (which is the zero of the denominator) is the same for the two expressions. Equality of the integrands is not required, but since we will be able to find systematic solutions to this condition, we impose it. It translates into

$$
1-\frac{V_{\mathrm{eff}}(r, E)}{p_{\infty}^{2}}=\frac{B(r) \mu^{2}}{p_{\mathrm{eff}}^{2}}\left(\frac{\mathcal{E}_{\mathrm{eff}}^{2}}{\mu^{2} A(r)}-1\right) .
$$

This expression can, after imposing $\mathcal{E}_{\text {eff }}^{2}=\mu^{2}+p_{\text {eff }}^{2}=$ $\gamma^{2} \mu^{2}$, be written as

$$
1-\frac{V_{\mathrm{eff}}(r, E)}{p_{\infty}^{2}}=\frac{B(r)}{\gamma^{2}-1}\left(\frac{\gamma^{2}}{A(r)}-1\right) .
$$

It is clear at this stage that we should not be able to find solutions for the metric functions $A(r)$ and $B(r)$ that are independent of $\gamma$, and they will, therefore, (utilizing the above identification) also depend on the effective energy. But if our objective is to identify a class of metrics that reproduce the scattering angle of the actual two-body problem using an EOB formalism, there is nothing to prevent us from pursuing this approach. Indeed, the only observable information we have at our disposal from the amplitude side is the scattering angle, and all remaining dynamics must be extracted from it. So the condition (29) fulfils our requirement. Using our parametrization for the metric coefficients in (18), this becomes a polynomial equation of sixth order in $h(r)$

$$
\begin{aligned}
& \left(h(r)+\frac{\gamma-1}{\gamma+1}\right)\left(h(r)+\frac{\gamma+1}{\gamma-1}\right)(1+h(r))^{4} \\
& =(1-h(r))^{2}\left(1+\frac{E^{2}}{\left(\gamma^{2}-1\right) M^{2}} \frac{V_{\text {eff }}(r, E)}{\nu^{2} M^{2}}\right) .
\end{aligned}
$$

This equation can always be solved in perturbation theory with $h(r)=\sum_{n \geq 1} h_{n}(G M / r)^{n}$ for any perturbatively expanded effective potential $V_{\text {eff }}=-\sum_{n \geq 1} f_{n}\left(G_{N} M / r\right)^{n}$. It is clear that if we had not used the simplifying ansatz (18) we would have, at each new order in $G_{N}$, two new metric coefficients to fit for each new condition from the scattering angle, allowing a large degree of freedom in the parametrization of the effective metric.

It is instructive to analyse in detail the first postMinkowskian approximation. Solving perturbatively for the coefficients $h_{n}$ in $h(r)=\sum_{n \geq 1} h_{n}(G M / r)^{n}$, we obtain

$$
\begin{gathered}
h_{1}=\frac{1}{2} \frac{E}{M}, \\
h_{2}=-\frac{3\left(5 \gamma^{2}-1\right)}{8\left(2 \gamma^{2}-1\right)}\left(1-\frac{M}{E}\right)\left(\frac{E}{M}\right)^{2},
\end{gathered}
$$

at the next order we split the expression for $h_{3}=h_{3}^{\text {cons }}+$ $h_{3}^{\mathrm{RR}}$ into a conservative part

$$
\begin{aligned}
h_{3}^{\text {cons }}= & \left(\frac{811 \gamma^{6}-224 \gamma^{5}-1665 \gamma^{4}-288 \gamma^{3}+659 \gamma^{2}+200 \gamma-45}{48\left(1-2 \gamma^{2}\right)^{2}\left(\gamma^{2}-1\right)}-\frac{\gamma\left(14 \gamma^{2}+25\right)}{6(\gamma-1)\left(2 \gamma^{2}-1\right)} \frac{M}{E}\right)\left(1-\frac{M}{E}\right)\left(\frac{E}{M}\right)^{3} \\
& -\frac{(\gamma+1)\left(4 \gamma^{4}-12 \gamma^{2}-3\right)}{2\left(\gamma^{2}-1\right)^{\frac{3}{2}}\left(2 \gamma^{2}-1\right)} \operatorname{arccosh}(\gamma)\left(1-\frac{M^{2}}{E^{2}}\right)\left(\frac{E}{M}\right)^{3},
\end{aligned}
$$

and a radiation-reaction part

$$
h_{3}^{\mathrm{RR}}=\left(2 \gamma^{2}-1\right)\left(\frac{\gamma\left(2 \gamma^{2}-3\right) \operatorname{arccosh}(\gamma)}{4(\gamma-1)^{3}(\gamma+1)^{2}}-\frac{(\gamma+1)\left(5 \gamma^{2}-8\right)}{12\left(\gamma^{2}-1\right)^{5 / 2}}\right)\left(1-\frac{M^{2}}{E^{2}}\right)\left(\frac{E}{M}\right)^{3} .
$$


One can argue whether the radiation-reaction terms $h_{3}^{\mathrm{RR}}$ should be included here. We have kept them because they are needed to produce the correct scattering angle in the high-energy limit.

In the probe limit, $\nu \rightarrow 0$, and the total energy $E$ in (4) becomes the total mass $M$. Up to third post-Minkowskian order, and including the radiation-reaction contributions, we find that the corrections $h_{2}$ and $h_{3}$ all vanish as $(E-M)$. We thus recover the Schwarzschild solution in isotropic coordinates since

$$
\lim _{\nu \rightarrow 0} h_{1}=\frac{1}{2} ; \quad \lim _{\nu \rightarrow 0} h_{i}=0 \quad \text { for } i=2,3 .
$$

Because the $f_{i}$ coefficients in (14)-(16) are proportional to $\mu^{2}=\nu^{2} M^{2}$, the effective potential has an overall factor of $\nu^{2}$ and it is convenient to separate it out by defining $V_{\text {eff }}^{\text {probe }}(r, M)$ through $V_{\text {eff }}(r, E) \equiv \nu^{2} V_{\text {eff }}^{\text {probe }}(r, M)+O\left(\nu^{3}\right)$. Since, furthermore, $p_{\infty}^{2}=M^{2} \nu^{2}\left(\gamma^{2}-1\right)+O\left(\nu^{3}\right)$, we of course also recover the probe potential for the Schwarzschild metric in isotropic coordinates,

$$
\begin{aligned}
V_{\mathrm{eff}}^{\mathrm{probe}}(r, M)= & M^{2}\left(\gamma^{2}-1\right)-M^{2}\left(1+\frac{G_{N} M}{2 r}\right)^{4} \\
& \times\left(\gamma^{2}\left(\frac{1+\frac{G_{N} M}{2 r}}{1-\frac{G_{N} M}{2 r}}\right)^{2}-1\right) .
\end{aligned}
$$

The effective energy function in isotropic coordinates that we propose here corresponds to

$\mathcal{E}_{\mathrm{eff}}^{2}=\frac{\left(1-h(r)^{2}\right.}{1+h(r))}\left[\mu^{2}+\frac{J_{\mathrm{eff}}^{2}}{r^{2}(1+h(r))^{4}}+\frac{p_{r}^{2}}{(1+h(r))^{4}}\right]$,

which in the probe limit becomes

$$
\left(\mathcal{E}_{\text {eff }}^{\text {probe }}\right)^{2}=\left(\frac{1-\frac{G_{N} M}{2 r}}{1+\frac{G_{N} M}{2 r}}\right)^{2}\left[\mu^{2}+\frac{p^{2}}{\left(1+\frac{G_{N} M}{2 r}\right)^{4}}\right],
$$

thus reproducing the Schwarzschild Hamiltonian given in Eq. (77) of [37].

So far, we have managed to find a simple effective EOB metric $g_{\mu \nu}^{\text {eff }}$ which correctly reproduces the scattering of two masses up to third post-Minkowskian order. The main use of an EOB metric is in the pseudoelliptic regime of bound orbits where the total energy (minus rest mass) is negative, and we now briefly consider the use of the metric $g_{\mu \nu}^{\text {eff }}$ in this regime.

An obvious first check of the metric would be to confirm that it reproduces the periastron shift of bound orbits to second order in the post-Minkowskian expansion. Clearly, to first post-Minkowskian order, the motion is Newtonian with a $1 / r$ potential and closed orbits. Adding to this the second-order solution for $h(r)$,

$$
h(r)=\frac{G_{N} E}{2 r}+\frac{3 G_{N}^{2}\left(5 \gamma^{2}-1\right) E(E-M)}{8\left(2 \gamma^{2}-1\right) r^{2}},
$$

it is a straightforward exercise to compute the periastron shift $\Delta \Phi$ from the EOB metric to this order in $G_{N}$. The result is

$$
\Delta \Phi=\frac{3 \pi G_{N}^{2} M^{2} \mu^{2}}{2 J^{2}}\left(\frac{E}{M}\right)\left(5 \gamma^{2}-1\right),
$$

which agrees with the computation of Ref. [38] where it was derived by analytic continuation from the scattering parameters. In the limit $E \simeq M$ and $\gamma \simeq 1$ it agrees with the classic result of Robertson for the two-body problem to that order (see Chapter 8.6 of [39]).

Finally, we can see how, conversely, the energy map (6) emerges in the present setting. We start with our condition (28) which imposes the correct scattering angle of the effective theory. We now keep $\mathcal{E}_{\text {eff }}, p_{\text {eff }}^{2}$, and $\mu$ a priori unrelated and analyze the condition order-by-order in the coupling $G_{N}$. To first post-Minkowskian order it reads

$$
\begin{aligned}
& \frac{\mu^{2}+p_{\mathrm{eff}}^{2}-\mathcal{E}_{\mathrm{eff}}^{2}}{p_{\mathrm{eff}}^{2}}+\left(\frac{f_{1}}{p_{\infty}^{2}}+\frac{4 h_{1}}{p_{\mathrm{eff}}^{2}}\left(\mu^{2}-2 \mathcal{E}_{\mathrm{eff}}^{2}\right)\right) \frac{G_{N} M}{r} \\
& +O\left(G_{N}^{2}\right)=0 .
\end{aligned}
$$

To zeroth order in $G_{N}$ we obtain the free particle relation $\mathcal{E}_{\text {eff }}^{2}=p_{\text {eff }}^{2}+\mu^{2}$. To order $G_{N}$ we next get, after making use of the leading-order relation and after inserting the expressions for $f_{1}$ from (14) and $h_{1}$ from (31),

$$
\frac{\mathcal{E}_{\text {eff }}}{\mu}=\sqrt{\frac{f_{1}-4 p_{\infty}^{2} h_{1}}{f_{1}-8 p_{\infty}^{2} h_{1}}}=\gamma=\frac{E^{2}-m_{1}^{2}-m_{2}^{2}}{2 m_{1} m_{2}},
$$

which is the energy map (6). From order $G_{N}^{2}$ and up this relationship is automatically satisfied by the condition (28).

\section{COMPARISON WITH EARLIER APPROACHES}

It is interesting to observe that the full leading-order metric we deduced above is not of Schwarzschild form but rather has the total mass $M=m_{1}+m_{2}$ replaced by total energy $E$, with

$$
h(r)=\sum_{n \geq 1} \hat{h}_{n}(\gamma, M / E)\left(\frac{G_{N} E}{r}\right)^{n},
$$

so that, to first post-Minkowskian order,

$$
\begin{aligned}
& A(r)=\left(\frac{1-\frac{G_{N} E}{2 r}}{1+\frac{G_{N} E}{2 r}}\right)^{2}+\mathcal{O}\left(G_{N}^{2}\right) \\
& B(r)=\left(1+\frac{G_{N} E}{2 r}\right)^{4}+\mathcal{O}\left(G_{N}^{2}\right) .
\end{aligned}
$$


While this energy-dependent metric may appear as an intuitively appealing effective metric for the postMinkowskian problem to this order, it seems to contradict the observation that to first order in the post-Minkowskian expansion the effective metric can be chosen to be exactly of Schwarzschild form [1]. The resolution is as follows. Our condition for the effective metric $g_{\mu \nu}^{\text {eff }}$ is that it solves the condition (20). As we have noted above, this leads us to solutions for the effective metric that are energy dependent. Instead, the conventional EOB formalism modifies the mass-shell condition in an alternative manner, replacing Eq. (20) by

$$
g_{\mathrm{eff}}^{\alpha \beta} \partial_{\alpha} \mathcal{S} \partial_{\beta} \mathcal{S}=\mu^{2}+Q,
$$

where the function $Q$ absorbs all terms higher than quadratic in the momenta. Both prescriptions correct for the fact that away from Minkowskian infinity we cannot insist on a purely quadratic equation in $\mathcal{E}_{\text {eff }}$. The analysis based on Eq. (45) in isotropic coordinates has first been performed in Ref. [3]. Imposing the usual angular momentum map $J=J_{\text {eff }}$ the condition of correct scattering angle must then read, in our notation,

$$
p_{\mathrm{eff}}^{2}+W(R)=\bar{B}(R)\left(\frac{\mathcal{E}_{\mathrm{eff}}^{2}}{\bar{A}(R)}-\mu^{2}-Q\right),
$$

where the functions $\bar{A}$ and $\bar{B}$ correspond to the Schwarzschild metric,

$$
\bar{A}(R)=\left(\frac{1-\frac{G_{N} M}{2 R}}{1+\frac{G_{N} M}{2 R}}\right)^{2} ; \quad \bar{B}(R)=\left(1+\frac{G_{N} M}{2 R}\right)^{4},
$$

and there is a rescaled three-momentum

$$
\mathbf{P}^{2}=P_{\infty}^{2}+W(R) .
$$

Comparing with the actual kinematical relation Eq. (11) of the two-body problem this allows us to identify

$$
\mathbf{P}^{2}=\frac{p_{\mathrm{eff}}^{2}}{p_{\infty}^{2}} p^{2}=\left(\frac{E}{M}\right)^{2} p^{2}
$$

and

$$
W(R)=-\frac{p_{\mathrm{eff}}^{2}}{p_{\infty}^{2}} V_{\mathrm{eff}}=-\left(\frac{E}{M}\right)^{2} V_{\mathrm{eff}}(r, E) .
$$

The two isotropic coordinates are related by $R=r \times$ $(M / E)$ and, as we see from Eq. (49), this is part of the canonical transformation

$$
\left(R, P_{R}\right)=\left(r \frac{M}{E}, p_{r} \frac{E}{M}\right)
$$

Expanding the potential $W(R)=\sum_{n \geq 1} \mu^{2} w_{n}\left(G_{N} M / R\right)^{n}$ as in Ref. [3] in terms of coefficients $w_{i}$ and after taking into account the relation between the two radii $r$ and $R$, we find the identification

$$
w_{i}=\frac{f_{i}}{\mu^{2}}\left(\frac{M}{E}\right)^{n-2} .
$$

Plugging in the coefficients $f_{i}$ one readily recovers the $w_{i}$ of Ref. [3] for $i=1,2$. Finally, rewriting the condition for the metric and $Q$ in the form

$$
1-\frac{V_{\mathrm{eff}}(r, E)}{p_{\infty}^{2}}=\frac{\bar{B}(R)}{p_{\mathrm{eff}}^{2}}\left(\frac{\mathcal{E}_{\mathrm{eff}}^{2}}{\bar{A}(R)}-\mu^{2}-Q\right),
$$

we can immediately compare with our (28). This gives

$$
\bar{B}(R)\left(\frac{\mathcal{E}_{\mathrm{eff}}^{2}}{\bar{A}(R)}-\mu^{2}-Q\right)=B(r)\left(\frac{\mathcal{E}_{\mathrm{eff}}^{2}}{A(r)}-\mu^{2}\right),
$$

where

$$
Q=\mu^{2} \sum_{n \geq 2} q_{n}\left(\frac{G_{N} M}{R}\right)^{n},
$$

Because both expressions yield the correct scattering angle, we should recover the $Q$ function from Ref. [3]. Indeed, inserting the Schwarzschild metric functions $\bar{A}$ and $\bar{B}$ and converting our $r$-coordinate to $R$ by the above canonical transformation, we obtain

$$
h(R)=\sum_{n \geq 1} h_{n}\left(\frac{M}{E}\right)^{n}\left(\frac{G_{N} M}{R}\right)^{n} .
$$

Expanding (54) and using that $h_{1}=E /(2 M)$ we get

$$
q_{2}=4\left(2 \gamma^{2}-1\right)\left(\frac{M}{E}\right)^{2} \times h_{2},
$$

which after using (32) reproduces the result given in Eq. (3.33) of Ref. [3]. Next, expanding (54) in $G_{N}$ and using the fact that $h$ starts at order $G_{N}$ gives

$$
\begin{aligned}
& \mathcal{E}_{\text {eff }}^{2} \sum_{n \geq 0} \sum_{p=0}^{\min (n, 6)} \frac{(n-p+1) 6 !}{p !(6-p) !}\left(\left(\frac{G_{N} M}{R}\right)^{n}-h(r)^{n}\right) \\
& =\mu^{2} \sum_{n \geq 2} q_{n}\left(\frac{G_{N} M}{R}\right)^{n} .
\end{aligned}
$$

Finally, using 


$$
h(R)^{n}=\sum_{m \geq n} \sum_{\substack{r_{1}+\cdots+r_{n}=m \\ r_{i} \geq 1}} \prod_{i=1}^{n} h_{r_{i}}\left(\frac{M}{E}\right)^{m}\left(\frac{G_{N} M}{R}\right)^{m},
$$

we have

$$
\begin{aligned}
q_{n}= & \gamma^{2} \sum_{p=0}^{\min (n, 6)} \frac{(n-p+1) 6 !}{p !(6-p) !}-\sum_{m=1}^{n} \sum_{p=0}^{\min (m, 6)} \frac{(m-p+1) 6 !}{p !(6-p) !} \\
& \times \sum_{r_{1}+\cdots+r_{m}=n} \prod_{\substack{r_{i \geq 1} \\
r_{i=1}}}^{n} h_{r_{i}}\left(\frac{M}{E}\right)^{n},
\end{aligned}
$$

which shows how to express the $q_{i}$-coefficients in terms of the $h_{i}$-coefficients of this paper.

To summarize this part: We have shown the equivalence between our remodeled EOB formalism in isotropic coordinates and the conventionally used formalism that separates out all nonquadratic energy-momentum terms in a function $Q$ which is added to the mass-shell condition. A canonical transformation distinguishes our formulation, which keeps the impact parameter $b$ fixed in the angular momentum map, from the conventional one. This choice of canonical coordinates allows us to immediately match the kinematical relation from amplitude computations with the EOB kinematics of the reduced problem. Additionally, we argued that it is far simpler to solve for the effective metric directly, without introducing such an auxiliary function $Q$ that parametrizes the deviations of the effective metric from the one of Schwarzschild. Expanding our solution around the Schwarzschild metric, we recover the $Q$-function of the literature, thus demonstrating the equivalence. The purpose of our remodeling has been to avoid this adding and subtraction of terms that are the origin of the $Q$-function. This seems to not be needed and one can instead work directly with the energy-dependent metric.

\section{CONCLUSIONS}

With a fresh look at the EOB formalism in the light of modern amplitude calculations for gravity, we have considered a modification of the conventionally phrased formalism which is not based upon an expansion around the static Schwarzschild metric. Instead, with a rather general assumption about the desired form of the effective one-body metric in isotropic coordinates, we have proposed a formulation where the metric coefficients are solved order-by-order from the scattering angles as computed from amplitudes. Crucial for this to come out in such a simple form has been the use of isotropic coordinates and an angular momentum map that differs from the one originally proposed. An interesting consequence is that we remain entirely within a metric framework, with no corrections terms needed, at least up to third post-Minkowskian order. The one principle that we have used to determine the effective metric is to equate the integrands of, on one side, the expression for the relativistic kinematics in isotropic coordinates and, on the other side, the expression based on the effective metric. In the probe limit we recover the Schwarzschild metric in isotropic coordinates and at any mass range our effective metric produces the correct scattering angle up to third post-Minkowskian order. We have also verified that the periastron shift at second postMinkowskian order is correctly reproduced. Finally, we have compared the above proposal with the conventional formalism and pointed out where differences appear even though both approaches reproduce correctly the observable quantities.

\section{ACKNOWLEDGMENTS}

We thank Andrea Cristofoli, Thibault Damour, and Justin Vines for very helpful discussions. The research of P.V. has received funding from the ANR Grant No. "Amplitudes" ANR-17- CE31-0001-01, and the ANR Grant No. "SMAGP" ANR-20-CE40-0026-01 and is partially supported by Laboratory of Mirror Symmetry NRU HSE, RF Government Grant Agreement No. 14.641.31.0001. The work of P. H. D. was supported in part by DFF Grant No. 0135-00089A.
[1] T. Damour, Gravitational scattering, Post-Minkowskian approximation and effective one-body theory, Phys. Rev. D 94, 104015 (2016).

[2] T. Damour, High-energy gravitational scattering and the general relativistic two-body problem, Phys. Rev. D 97, 044038 (2018).

[3] T. Damour, Classical and quantum scattering in postMinkowskian gravity, Phys. Rev. D 102, 024060 (2020).
[4] N. E. J. Bjerrum-Bohr, P. H. Damgaard, G. Festuccia, L. Planté, and P. Vanhove, General Relativity from Scattering Amplitudes, Phys. Rev. Lett. 121, 171601 (2018).

[5] C. Cheung, I. Z. Rothstein, and M. P. Solon, From Scattering Amplitudes to Classical Potentials in the Post-Minkowskian Expansion, Phys. Rev. Lett. 121, 251101 (2018).

[6] D. A. Kosower, B. Maybee, and D. O'Connell, Amplitudes, observables, and classical scattering, J. High Energy Phys. 02 (2019) 137. 
[7] Z. Bern, C. Cheung, R. Roiban, C. H. Shen, M. P. Solon, and M. Zeng, Scattering Amplitudes and the Conservative Hamiltonian for Binary Systems at Third Post-Minkowskian Order, Phys. Rev. Lett. 122, 201603 (2019); Z. Bern, C. Cheung, R. Roiban, C. H. Shen, M. P. Solon, and M. Zeng, Black hole binary dynamics from the double copy and effective theory, J. High Energy Phys. 10 (2019) 206.

[8] A. Antonelli, A. Buonanno, J. Steinhoff, M. van de Meent, and J. Vines, Energetics of two-body Hamiltonians in postMinkowskian gravity, Phys. Rev. D 99, 104004 (2019).

[9] A. Cristofoli, N. E. J. Bjerrum-Bohr, P. H. Damgaard, and P. Vanhove, Post-Minkowskian Hamiltonians in general relativity, Phys. Rev. D 100, 084040 (2019).

[10] G. Kälin and R. A. Porto, From boundary data to bound states, J. High Energy Phys. 01 (2020) 072.

[11] N. E. J. Bjerrum-Bohr, A. Cristofoli, and P. H. Damgaard, Post-Minkowskian scattering angle in Einstein gravity, J. High Energy Phys. 08 (2020) 038.

[12] A. Cristofoli, P. H. Damgaard, P. Di Vecchia, and C. Heissenberg, Second-order Post-Minkowskian scattering in arbitrary dimensions, J. High Energy Phys. 07 (2020) 122.

[13] J. Parra-Martínez, M. S. Ruf, and M. Zeng, Extremal black hole scattering at $\mathcal{O}\left(G^{3}\right)$ : Graviton dominance, eikonal exponentiation, and differential equations, J. High Energy Phys. 11 (2020) 023.

[14] P. Di Vecchia, C. Heissenberg, R. Russo, and G. Veneziano, Universality of ultra-relativistic gravitational scattering, Phys. Lett. B 811, 135924 (2020).

[15] T. Damour, Radiative Contribution to classical gravitational scattering at the third order in $G$, Phys. Rev. D 102, 124008 (2020).

[16] P. Di Vecchia, C. Heissenberg, R. Russo, and G. Veneziano, Radiation reaction from soft theorems, Phys. Lett. B 818, 136379 (2021).

[17] Z. Bern, J. Parra-Martinez, R. Roiban, M. S. Ruf, C. H. Shen, M. P. Solon, and M. Zeng, Scattering Amplitudes and Conservative Binary Dynamics at $\mathcal{O}\left(G^{4}\right)$, Phys. Rev. Lett. 126, 171601 (2021).

[18] P. Di Vecchia, C. Heissenberg, R. Russo, and G. Veneziano, The eikonal approach to gravitational scattering and radiation at $\mathcal{O}\left(G^{3}\right)$, J. High Energy Phys. 07 (2021) 169.

[19] N. E. J. Bjerrum-Bohr, P. H. Damgaard, L. Planté, and P. Vanhove, Classical gravity from loop amplitudes, Phys. Rev. D 104, 026009 (2021).

[20] N. E. J. Bjerrum-Bohr, P. H. Damgaard, L. Planté, and P. Vanhove, The amplitude for classical gravitational scattering at third post-Minkowskian order, arXiv:2105.05218.

[21] D. Bini, T. Damour, and A. Geralico, Radiative contributions to gravitational scattering, Phys. Rev. D 104, 084031 (2021).

[22] Y. F. Bautista, A. Guevara, C. Kavanagh, and J. Vines, From scattering in black hole backgrounds to higher-spin amplitudes: Part I, arXiv:2107.10179.
[23] A. Cristofoli, R. Gonzo, D. A. Kosower, and D. O'Connell, Waveforms from amplitudes, arXiv:2107.10193.

[24] E. Herrmann, J. Parra-Martinez, M. S. Ruf, and M. Zeng, Gravitational Bremsstrahlung from Reverse Unitarity, Phys. Rev. Lett. 126, 201602 (2021).

[25] E. Herrmann, J. Parra-Martinez, M. S. Ruf, and M. Zeng, Radiative classical gravitational observables at $\mathcal{O}\left(G^{3}\right)$ from scattering amplitudes, arXiv:2104.03957.

[26] S. Mougiakakos, M. M. Riva, and F. Vernizzi, Gravitational Bremsstrahlung in the post-Minkowskian effective field theory, Phys. Rev. D 104, 024041 (2021).

[27] G. U. Jakobsen, G. Mogull, J. Plefka, and J. Steinhoff, Classical Gravitational Bremsstrahlung from a Worldline Quantum Field Theory, Phys. Rev. Lett. 126, 201103 (2021).

[28] P. H. Damgaard, L. Planté, and P. Vanhove, On an exponential representation of the gravitational S-matrix, arXiv: 2107.12891.

[29] A. Brandhuber, G. Chen, G. Travaglini, and C. Wen, Classical gravitational scattering from a gauge-invariant double copy, arXiv:2108.04216.

[30] A. Buonanno and T. Damour, Effective one-body approach to general relativistic two-body dynamics, Phys. Rev. D 59, 084006 (1999).

[31] A. Buonanno and T. Damour, Transition from inspiral to plunge in binary black hole coalescences, Phys. Rev. D 62, 064015 (2000).

[32] T. Damour, P. Jaranowski, and G. Schaefer, On the determination of the last stable orbit for circular general relativistic binaries at the third post-Newtonian approximation, Phys. Rev. D 62, 084011 (2000).

[33] T. Damour, Coalescence of two spinning black holes: An effective one-body approach, Phys. Rev. D 64, 124013 (2001).

[34] A. Buonanno, Y. Chen, and T. Damour, Transition from inspiral to plunge in precessing binaries of spinning black holes, Phys. Rev. D 74, 104005 (2006).

[35] J. Vines, Scattering of two spinning black holes in postMinkowskian gravity, to all orders in spin, and effectiveone-body mappings, Classical Quantum Gravity 35, 084002 (2018).

[36] J. Vines, J. Steinhoff, and A. Buonanno, Spinning-black-hole scattering and the test-black-hole limit at second postMinkowskian order, Phys. Rev. D 99, 064054 (2019).

[37] P. Jaranowski and G. Schaefer, Third post-Newtonian higher order ADM Hamilton dynamics for two-body point mass systems, Phys. Rev. D 57, 7274 (1998); Erratum, Phys. Rev. D 63, 029902 (2000).

[38] G. Kälin and R. A. Porto, From boundary data to bound states. Part II. Scattering angle to dynamical invariants (with twist), J. High Energy Phys. 02 (2020) 120.

[39] S. Weinberg, Gravitation and Cosmology: Principles and Applications of the General Theory of Relativity (John Wiley \& Sons, New York, 1972). 